\title{
Clinical value of ultrasonic imaging in diagnosis of hypopharyngeal cancer with cervical lymph node metastasis
}

\author{
GUZI WANG ${ }^{1}$, XIAOGUANG LI ${ }^{1}$, LI LI ${ }^{1}$, DAYU LIU ${ }^{2}$, RUIJIE SUN ${ }^{2}$, QIU ZHANG ${ }^{1}$, \\ CHENCHEN GENG ${ }^{1}$, HAITONG GONG $^{1}$ and XIAOQIAN GAO ${ }^{1}$
}

\author{
Departments of ${ }^{1}$ Ultrasound and ${ }^{2}$ Head and Neck Surgery, Qilu Hospital of Shandong University (Qingdao), \\ Qingdao, Shandong 266035, P.R. China
}

Received April 4, 2019; Accepted June 12, 2019

DOI: $10.3892 / \mathrm{ol} .2019 .10939$

\begin{abstract}
Clinical value of ultrasonic imaging in diagnosis of hypopharyngeal cancer with cervical lymph node metastasis was investigated. Eighty-nine patients who were diagnosed with hypopharyngeal cancer in Qilu Hospital of Shandong University (Qingdao) from January 2014 to June 2016 were retrospectively analyzed. Sixty-eight patients were diagnosed with hypopharyngeal cancer with cervical lymph node metastasis by pathological sections. Twenty-one patients did not have cervical lymph node metastasis. All the patients were diagnosed by palpation and ultrasound. The lymph node ultrasound images were quantified by computer, and the long/short diameter ratio, the maximum systolic velocity, blood flow resistance of the metastatic and non-metastatic patients were compared. The diagnostic efficacy of palpation and ultrasound was analyzed in the diagnosis of hypopharyngeal cancer with cervical lymph node metastasis. A correlation analysis was carried out between the image features of ultrasound and lymph node metastasis. The long/short diameter ratio, maximum systolic velocity and resistance index of patients with lymph node metastasis were significantly higher than those without lymph node metastasis, with a significant difference $(\mathrm{P}<0.05)$. Forty-one patients were diagnosed with lymph node metastasis by palpation, fifty-nine patients were diagnosed with lymph node metastasis by ultrasound. The sensitivity and diagnostic coincidence rate of ultrasound in diagnosis of hypopharyngeal cancer with cervical lymph node metastasis were significantly higher than those of palpation $(\mathrm{P}<0.05)$. Statistically significant differences were observed in lymph node internal echo types, medullary echo characteristics, envelope definition, and blood flow distribution characteristics between the metastasis group and the non-metastasis group $(\mathrm{P}<0.05)$. Lymph node
\end{abstract}

Correspondence to: Dr Guzi Wang, Department of Ultrasound, Qilu Hospital of Shandong University (Qingdao), 758 Hefei Road, Qingdao, Shandong 266035, P.R. China

E-mail: ufp45w@163.com

Key words: ultrasound, hypopharyngeal cancer, cervical lymph node metastasis, diagnostic value internal echo was heterogeneous. There was no medulla, and the disordered blood flow in the lymph node predicted lymph node metastasis. Preoperative ultrasound has a high diagnostic value in diagnosis of hypopharyngeal cancer with cervical lymph node metastasis. The diagnostic results of preoperative ultrasound can be used as a reference for the diagnosis and treatment of hypopharyngeal cancer with cervical lymph node metastasis.

\section{Introduction}

Hypopharyngeal cancer is a malignant tumor with poor prognosis in head and neck cancer. Its morbidity accounts for $3-5 \%$ of head and neck cancers (1). As the pathogenic location of hypopharyngeal cancer is concealed, the lesion tends to infiltrate and spread along the mucosa. The incidence of cervical lymph node metastasis is high. Cervical lymph node metastasis has already appeared in patients when they are diagnosed. Cervical lymph node metastasis is one of the factors that affect prognosis of patients with hypopharyngeal cancer $(2,3)$. Therefore, how to accurately diagnose and evaluate lymph node metastasis in patients with hypopharyngeal cancer has important clinical significance for patients with cervical lymph node metastasis of hypopharyngeal cancer (4).

Clinical examination of cervical lymph node metastasis has been carried out by palpation, but the accuracy of palpation is easily affected by factors, such as the experience of the examiner and the location and size of lymph nodes (5). For example, the lymph node has no obvious swelling and tenderness in patients with cervical lymph node metastasis of early hypopharyngeal cancer. The texture of the lymph node is soft and the size is small, so it is difficult to be found by palpation (6). Ultrasound, as a non-invasive, economical and convenient method, has been used to diagnose many diseases in clinic (7). Studies have shown that ultrasound has diagnostic value for hypopharyngeal cancer and that cervical lymph node metastasis missed by palpation can be found by ultrasound (8).

However, research reports on the diagnosis of regional lymph node metastasis of hypopharyngeal cancer are generally mentioned in comprehensive reports on head and neck tumors (9). The studies that specifically report lymph node metastasis of hypopharyngeal cancer are rare. Therefore, the results of pathological examination were used as the standard, 
the diagnostic value of ultrasonic imaging was investigated for lymph node metastasis of hypopharyngeal cancer, in order to provide proper plans for diagnosis of patients with hypopharyngeal cancer with lymph node metastasis.

\section{Patients and methods}

General data. Eighty-nine patients who were diagnosed with hypopharyngeal cancer in Qilu Hospital of Shandong University (Qingdao) (Qingdao, China) from January 2014 to June 2016 were retrospectively analyzed, including 51 male patients and 38 female patients, with an average age of $54.1 \pm 2.5$ years. Sixty-eight patients were diagnosed with hypopharyngeal cancer with cervical lymph node metastasis by pathological sections. Twenty-one patients did not have cervical lymph node metastasis (Table I).

Inclusion criteria: Patients who were diagnosed with hypopharyngeal cancer by pathological examination. Exclusion criteria: Patients with severe liver dysfunction and kidney dysfunction; patients with hypopharyngeal cancer and other cancers; patients with severe infection; patients who did not accept pathological diagnosis; patients with cognitive disorder or communication disorder; patients who would not cooperate with the experiment. All the patients and their family members agreed to participate in the experiment and signed informed consent forms. This experiment was approved by the Ethics Committee of Qilu Hospital of Shandong University (Qingdao).

\section{Detection methods}

Palpation. The diagnosis of metastatic lymph node was made by experienced doctors in a subjective way. At present, only the order and description can be specified for palpation. All the patients were diagnosed by palpation, which was carried out by multiple doctors who had rich clinical experience to reduce bias. When the doctors carried out palpation, they gently touched the neck of the patients with their hands in up to down manner. The key area was the lymph node chain of the internal jugular vein, for lymph nodes in the II-IV area. The presence and absence of lymphadenopathy and the size, hardness, and mobility of the lymph nodes were diagnosed and assessed.

Ultrasound. ATL-HDI 5000 color Doppler ultrasound equipment (Philips) was used to diagnose the patients. The probe frequency was $7 \mathrm{MHz}$, the aliasing speed of the color flow was adjusted to $8 \mathrm{~cm} / \mathrm{sec}$, the color gain was adjusted to $80-85 \%$. The patients were in supine position, with neck exposed and raised with a pillow. When the patients were examined, the head was turned to the side that was not examined, the lymph nodes were scanned by seven-point method (10) of American Joint Committee on Cancer. The scanning range was from the area behind the ear to supraclavicular fossa, the longitudinal and transverse examinations were carried out. The location, shape, size, internal echo and envelope of the lymph nodes were recorded. The blood flow of the lymph nodes was observed and the lymph nodes were divided into several types, including non-blood flow type, central type, peripheral type and hybrid type. However, the artificial interpretation of cervical lymph node ultrasound images is subjective, so the analysis of lymph node ultrasound images was performed by computer. The diagnostic criteria for malignant lymph nodes were detailed previously $(11,12)$.
Table I. General data.

Patients with hypopharyngeal

Items cancer $(n=89)$

Sex

Male

$51(57.30)$

Female

Age (years)

$\geq 54$

$45(50.56)$

$<54$

BMI

$\geq 21$

37 (41.57)

$<21$

Educational level

$\geq$ Middle school

$<$ Middle school

Presence and absence of cervical

lymph node metastasis

Presence

$68(76.40)$

Absence

Liver function indicators

Serum total protein $(\mathrm{g} / \mathrm{l})$

$7142 \pm 2.33$

Alanine aminotransferase $(\mu \mathrm{mol} / \mathrm{l})$

$28.34 \pm 4.58$

Total bilirubin $(\mu \mathrm{mol} / \mathrm{l})$

$11.27 \pm 2.10$

Renal function indicators $(\mu \mathrm{mol} / \mathrm{l})$

Creatinine

$59.59 \pm 4.11$

Serum urea

$5.19 \pm 0.76$

Uric acid

$272.66 \pm 11.74$

Observation indicators. i) The long/short-diameter ratio of the characteristic lymph nodes, the maximum systolic velocity and the blood flow resistance index of the ultrasound images were compared between the two groups. ii) The sensitivity, specificity, diagnostic accuracy, positive predictive value and negative predictive value of palpation and ultrasound were calculated and compared in the diagnosis of hypopharyngeal cancer with cervical lymph node metastasis. iii) An association analysis was carried out between the imaging features of ultrasound and lymph node metastasis.

Statistical analysis. SPSS 19.0 (IBM) statistical software was used to analyze the experimental data. Chi-square test was used for the enumeration data. The association between the imaging features and lymph node metastasis was analyzed by Logistic regression analysis. Student's t test was used for quantitative parameters of ultrasound features analysis. $\mathrm{P}<0.05$ was considered to be statistically significant.

\section{Results}

Comparison of ultrasound image features in patients with and without metastasis. The long/short diameter ratio, maximum systolic velocity and resistance index of patients with lymph 
Table II. Quantitative parameters of ultrasound features.

\begin{tabular}{lcrrr}
\hline Ultrasonic features & Metastasis $(\mathrm{n}=68)$ & Non-metastasis $(\mathrm{n}=21)$ & $\mathrm{t}$ value & P-value \\
\hline Long/short diameter ratio & $2.29 \pm 0.72$ & $1.52 \pm 0.63$ & 6.637 & $<0.001$ \\
Maximum systolic velocity & $34.89 \pm 8.31$ & $24.33 \pm 7.91$ & 7.590 & $<0.001$ \\
Resistance index & $0.72 \pm 0.10$ & $0.53 \pm 0.08$ & 12.23 & $<0.001$ \\
\hline
\end{tabular}

Table III. Diagnostic results of hypopharyngeal cancer with cervical lymph node metastasis diagnosed by palpation.

\begin{tabular}{lccc}
\hline Diagnostic results & Pathology (metastasis) & Pathology (non-metastasis) & Summation \\
\hline Diagnosis (metastasis) & 41 & 8 & 49 \\
Diagnosis (non-metastasis) & 27 & 13 & 40 \\
Summation & 68 & 21 & 89 \\
\hline
\end{tabular}

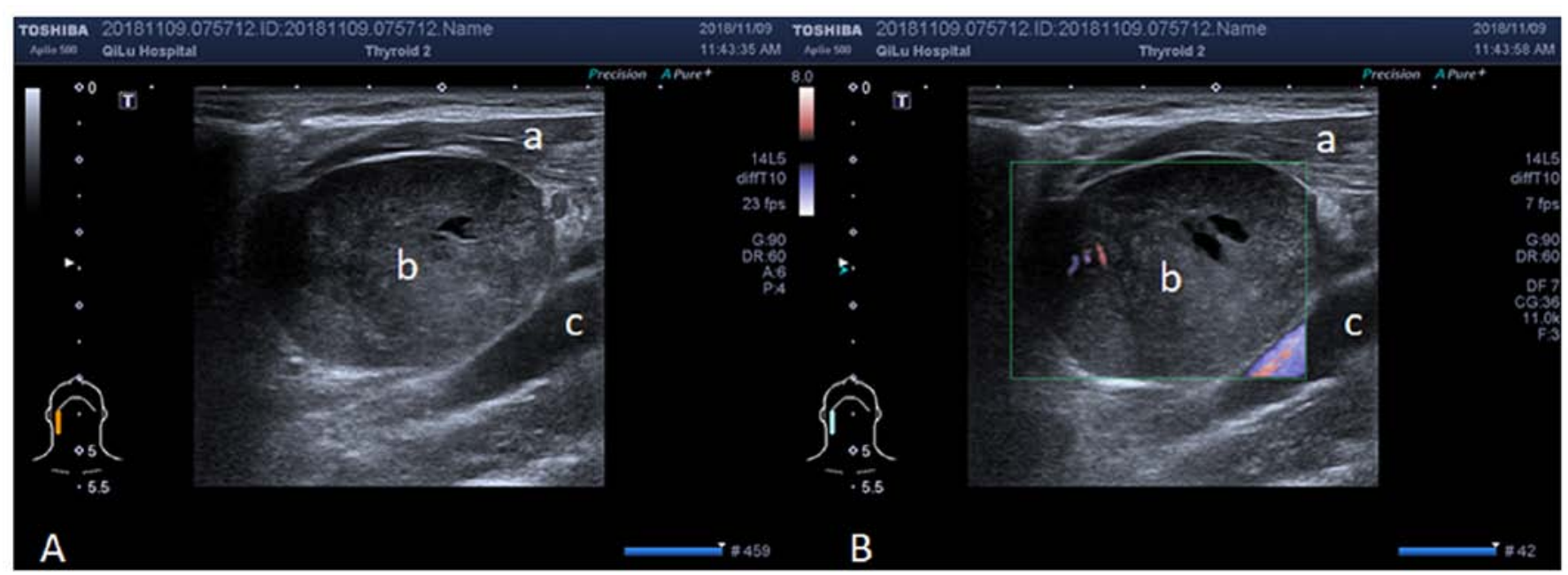

Figure 1. Ultrasound findings of abnormally enlarged lymph nodes in the neck. (A) Two-dimensional sonogram; (B) Color Doppler ultrasound. (a) Sternocleidomastoid muscle; (b) abnormally enlarged lymph node; (c) carotid artery.

node metastasis were significantly higher than those without lymph node metastasis, and the difference was statistically significant $(\mathrm{P}<0.05)$ (Table II).

Analysis of diagnostic value of palpation and ultrasound in hypopharyngeal cancer with cervical lymph node metastasis. Forty-one patients were diagnosed with lymph node metastasis by palpation, 59 patients were diagnosed for lymph node metastasis by ultrasound. The sensitivity, specificity, diagnostic coincidence rate, negative predictive value and positive predictive value of palpation were $69.29,61.90,60.67$ 32.5 and $83.67 \%$, respectively, in the diagnosis of hypopharyngeal cancer with cervical lymph node metastasis, and those of ultrasound were $86.76,80.95,85.39,65.38$ and $93.65 \%$, respectively. The sensitivity and diagnostic coincidence rate of ultrasound in diagnosis of hypopharyngeal cancer with cervical lymph node metastasis were significantly higher than those of palpation. The differences were statistically significant $(\mathrm{P}<0.05)$. The specificity, negative predictive value and positive predictive value of ultrasound were higher than those of palpation, but the differences were not statistically significant $(\mathrm{P}>0.05)$ (Tables III-V).

The relationship between imaging features of ultrasound and pathological findings. Statistically significant differences were observed in lymph node internal echo types, medullary echo characteristics, envelope definition, and blood flow distribution characteristics between the metastasis group and the non-metastasis group $(\mathrm{P}<0.05)$ (Table VI and Fig. 1).

Multivariate logistic regression analysis. Analysis was carried out for the diagnostic results of ultrasound. The results showed that lymph node internal echo was heterogeneous. There was no medulla, and the disordered blood flow in the lymph node predicted lymph node metastasis $(\mathrm{P}<0.001)$ (Table VII).

\section{Discussion}

Hypopharyngeal cancer is a refractory head and neck tumor, with malignant biological behavior, and it is prone to infiltration 
Table IV. Diagnostics of hypopharyngeal cancer with cervical lymph node metastasis by ultrasound.

\begin{tabular}{lccc}
\hline Diagnostic results & Pathology (metastasis) & Pathology (non-metastasis) & Summation \\
\hline Diagnosis (metastasis) & 59 & 4 & 63 \\
Diagnosis (non-metastasis) & 9 & 17 & 26 \\
Summation & 68 & 21 & 89 \\
\hline
\end{tabular}

Table V. Analysis of diagnostic efficacy of palpation and ultrasound in hypopharyngeal cancer with cervical lymph node metastasis $(\%)$.

\begin{tabular}{|c|c|c|c|c|}
\hline Diagnostic value & Palpation & Ultrasound & $\chi^{2}$ & P-value \\
\hline Sensitivity & $69.29 \%$ & $86.76 \%$ & 12.24 & $<0.001$ \\
\hline Specificity & $61.90 \%$ & $80.95 \%$ & 1.876 & 0.172 \\
\hline Diagnostic coincidence rate & $60.67 \%$ & $85.39 \%$ & 13.81 & $<0.001$ \\
\hline Negative predictive value & $32.5 \%$ & $65.38 \%$ & 0.284 & 0.594 \\
\hline Positive predictive value & $83.67 \%$ & $93.65 \%$ & 2.868 & 0.090 \\
\hline
\end{tabular}

Table VI. The relationship between imaging features of ultrasound and pathological findings.

\begin{tabular}{|c|c|c|c|c|}
\hline Imaging features & Metastasis $(\mathrm{n}=68)$ & Non-metastasis $(n=21)$ & $\chi^{2}$ & P-value \\
\hline Internal echo & & & 47.04 & $<0.001$ \\
\hline Homogeneous hypoecho & $8(11.76)$ & $19(90.48)$ & & \\
\hline Heterogeneous hypoecho & $60(88.24)$ & $2(9.52)$ & & \\
\hline Medulla & & & 75.62 & $<0.001$ \\
\hline No medulla & $63(92.65)$ & $1(4.76)$ & & \\
\hline Broadening, centering & $5(7.35)$ & $2(9.52)$ & & \\
\hline Threadiness, centering & 0 & $18(85.71)$ & & \\
\hline Envelope & & & 36.01 & $<0.001$ \\
\hline Clear & $15(22.06)$ & $20(95.24)$ & & \\
\hline Not clear & $53(77.94)$ & $1(4.76)$ & & \\
\hline The type of blood flow & & & 48.90 & $<0.001$ \\
\hline No blood flow & $10(14.71)$ & $7(33.33)$ & & \\
\hline Central regular blood flow & $4(5.88)$ & $14(66.67)$ & & \\
\hline Central disorderly blood flow & $27(39.71)$ & 0 & & \\
\hline Peripheral disorderly blood flow & $27(39.71)$ & 0 & & \\
\hline
\end{tabular}

Table VII. Multivariate regression analysis.

\begin{tabular}{lcrr}
\hline Ultrasound findings & Correlation index & Standard error & P-value \\
\hline Internal echo & 3.825 & 1.229 & $<0.001$ \\
Medulla deformation & 2.492 & 0.671 & $<0.001$ \\
Blood flow & 1.753 & 0.562 & $<0.001$ \\
\hline
\end{tabular}

and metastasis in mucosa (13). In recent years, although local control rate of hypopharyngeal cancer has increased with the development of medical technology, its 5-year survival rate is still $25-40 \%$ and is difficult to be improved (14). Therefore, how to make an effective diagnosis for hypopharyngeal cancer with cervical lymph node metastasis as soon as possible is an urgent problem to be solved. At present, in addition to palpation, there are other diagnostic methods for tumors in the throat, such as ultrasound, CT and MRI (15). However, MRI is expensive and there is radiation in $\mathrm{CT}$, thus these two methods 
are currently not popular (16). Ultrasound has advantages of being non-invasive, economical and convenient (17), so the diagnostic value of ultrasound was analyzed in hypopharyngeal cancer with lymph node metastasis.

In the present study, according to the results in the comparison of ultrasound image parameters, the long/short diameter ratio, the maximum systolic velocity and the resistance index of patients with lymph node metastasis were significantly higher than those without lymph node metastasis. The diagnostic efficacy of palpation and ultrasound was analyzed in hypopharyngeal cancer with lymph node metastasis. The results showed that the patients with lymph node metastasis diagnosed by ultrasound was significantly more than that of palpation $(\mathrm{P}<0.05)$. The sensitivity and diagnostic coincidence rate of ultrasound in hypopharyngeal cancer with cervical lymph node metastasis were significantly higher than those of palpation $(\mathrm{P}<0.05)$. Although the specificity, negative predictive value, and positive predictive value of ultrasound were higher than those of palpation, the differences were not statistically significant $(\mathrm{P}>0.05)$. Studies have reported that the sensitivity and specificity of ultrasound in cervical lymph node metastasis were higher than those of palpation, which confirmed the result of this study $(18,19)$. The relationship between the image features of ultrasound in lymph nodes and the pathological findings was analyzed. Statistically significant differences were observed in lymph node internal echo types, medullary echo characteristics, envelope definition, and blood flow distribution characteristics between the metastasis group and the non-metastasis group $(\mathrm{P}<0.05)$. Multivariate logistic regression analysis also showed that lymph node internal echo was heterogeneous, there was no medulla, and that the disordered blood flow in lymph nodes could indicate lymph node metastasis. It was reported that the diagnostic value of ultrasound in laryngeal cancer and hypopharyngeal cancer with cervical lymph node metastasis and demonstrated that lymph node internal echo was heterogeneous, there was no medulla or medulla decentered, and that the disordered signal of blood flow in lymph nodes could indicate lymph node metastasis (20). This result is consistent with the results of the present study. Furthermore, it was reported that increased blood flow signals around lymph nodes indicated the presence of lymph node metastasis, the reason is that the infiltration of cancer cells injures blood vessels of medulla, resulting in the residual of blood vessels of the tunica (21). This explains the abnormality of the blood flow signal. A report also indicated that the ultrasound image of cervical lymph node metastasis showed medullary structural disorders, and that blood flow showed the increase of peripheral blood flow signal and the increase of resistance index (22). This result is also consistent with the findings of this study.

In conclusion, cervical lymph node metastasis is an important independent prognostic factor for patients with hypopharyngeal cancer with cervical lymph node metastasis $(23,24)$. The diagnostic accuracy has important clinical value. This study showed that the preoperative ultrasound has diagnostic value for hypopharyngeal cancer with cervical lymph node metastasis and that the diagnosis result of preoperative ultrasound can be used as an important reference for the diagnosis and treatment of hypopharyngeal cancer with lymph node metastasis. However, further studies are still required.

\section{Acknowledgements}

Not applicable.

\section{Funding}

No funding was received.

\section{Availability of data and materials}

The datasets used and/or analyzed during the present study are available from the corresponding author on reasonable request.

\section{Authors' contributions}

GW wrote the manuscript. XL, RS and LL collected and analyzed the general data of patients. GW, DL and QZ recorded and analyzed ultrasound results. $\mathrm{CG}$ and $\mathrm{HG}$ contributed to statistical analysis. All authors read and approved the final manuscript.

\section{Ethics approval and consent to participate}

The study was approved by the Ethics Committee of Qilu Hospital of Shandong University (Qingdao) (Qingdao, China). Patients who participated in this study, had complete clinical data. Signed informed consents were obtained from the patients or the guardians.

\section{Patient consent for publication}

Not applicable.

\section{Competing interests}

The authors declare that they have no competing interests.

\section{References}

1. Kim JW, Kim MS, Kim SH, Kim JH, Lee CG, Kim GE and Keum KC: Definitive chemoradiotherapy versus surgery followed by adjuvant radiotherapy in resectable stage III/IV hypopharyngeal cancer. Cancer Res Treat 48: 45-53, 2016.

2. Chung EJ, Kim GW, Cho BK, Park HS and Rho YS: Pattern of lymph node metastasis in hypopharyngeal squamous cell carcinoma and indications for level VI lymph node dissection. Head Neck 38 (Suppl 1): E1969-1973, 2016.

3. Xing Y, Zhang J, Lin H, Gold KA, Sturgis EM, Garden AS, Lee JJ and William WN Jr: Relation between the level of lymph node metastasis and survival in locally advanced head and neck squamous cell carcinoma. Cancer 122: 534-545, 2016.

4. Wang Z, Ji W, Tang Q and Pan Z: Relationship among expression of the VEGF gene and MVD with cervical lymph node metastasis in laryngeal squamous cell carcinoma. Lin Chuang Er Bi Yan Hou Ke Za Zhi 18: 100-102, 2004 (In Chinese).

5. Liang H, Li A, Li Y, Cheng H, Zhao Q, Li J and Wang Q: A retrospective study of dual-energy CT for clinical detecting of metastatic cervical lymph nodes in laryngeal and hypopharyngeal squamous cell carcinoma. Acta Otolaryngol 135: 722-728, 2015.

6. Ni XG, Cheng RR, Lai SQ, Zhang L, He S, Zhang YM and Wang GQ: Value of narrow band imaging endoscopy in the detection of unknown primary site with cervical lymph node metastasis of squamous cell carcinoma. Zhonghua Zhong Liu Za Zhi 35: 698-702, 2013 (In Chinese). 
7. Richardson A, Gallos I,Dobson S, Campbell BK, Coomarasamy A and Raine-Fenning N: Accuracy of first-trimester ultrasound in diagnosis of tubal ectopic pregnancy in the absence of an obvious extrauterine embryo: Systematic review and meta-analysis. Ultrasound Obstet Gynecol 47: 28-37, 2016.

8. Arens C, Weigt J, Schumacher J and Kraft M: Ultrasound of the larynx, hypopharynx and upper esophagus. HNO 59: 145-154, 2011 (In German).

9. Buckley JG and MacLennan K: Cervical node metastases in laryngeal and hypopharyngeal cancer: A prospective analysis of prevalence and distribution. Head Neck 22: 380-385, 2000.

10. Prativadi R, Dahiya N, Kamaya A and Bhatt S: Chapter 5 ultrasound characteristics of benign vs. malignant cervical lymph nodes. Semin Ultrasound CT MR 38: 506-515, 2017.

11. Randolph G, Sacks B and Sr Baskin HJ: Ultrasound and mapping of neck lymph nodes. In: Thyroid Ultrasound and Ultrasound-Guided FNA. Baskin Sr. HJ, Duick DS and Levine RA (eds.) Springer, New York, NY, pp149-177, 2018.

12. Xia Y: Value of ultrasonography in differentiating malignant and benign superficial cervical lymph nodes. Zhongguo Yi Xue Ying Xiang Ji Shu 27: 45-48, 2011 (In Chinese).

13. Wu Z, Deng XY, Zeng RF, Su Y, Gu MF, Zhang Y, Xie CM and Zheng L: Analysis of risk factors for retropharyngeal lymph node metastasis in carcinoma of the hypopharynx. Head Neck 35: $1274-1277,2013$.

14. Wang J, Cui XB and Sun YH: Significance of induction chemotherapy in the treatment of locally advanced laryngeal cancer and hypopharyngeal cancer. Lin Chung Er Bi Yan Hou Tou Jing Wai Ke Za Zhi 30: 1548-1551, 2016 (In Chinese).

15. Mok G, Gauthier I, Jiang H, Huang SH, Chan K, Witterick IJ, O'Sullivan B, Waldron JN, Bayley AJ, Cho BC, et al: Outcomes of intensity-modulated radiotherapy versus conventional radiotherapy for hypopharyngeal cancer. Head Neck 37: 655-661, 2015.

16. Marioni G, Bertolin A, Giacomelli L, Marchese-Ragona R, Savastano M, Calgaro N, Marino F, De Filippis C and Staffieri A: Expression of the apoptosis inhibitor protein Survivin in primary laryngeal carcinoma and cervical lymph node metastasis. Anticancer Res 26: 3813-3817, 2006.
17. Leng XF, Zhu Y, Wang GP, Jin J, Xian L and Zhang YH: Accuracy of ultrasound for the diagnosis of cervical lymph node metastasis in esophageal cancer: a systematic review and meta-analysis. J Thorac Dis 8: 2146-2157, 2016.

18. van den Brekel MW, Stel HV, Castelijns JA, Nauta JJ, van der Waal I, Valk J, Meyer CJ and Snow GB: Cervical lymph node metastasis: assessment of radiologic criteria. Radiology 177: 379-384, 1990.

19. van den Brekel MW, Castelijns JA, Stel HV, Luth WJ, Valk J, van der Waal I and Snow GB: Occult metastatic neck disease: detection with US and US-guided fine-needle aspiration cytology. Radiology 180: 457-461, 1991.

20. Tang QF, Ji WY, Pan ZM, Zheng Y and Guan C: Expression of the metastasis-associated gene 1 in laryngeal squamous cell carcinoma: correlation with cervical lymph node metastasis. Zhonghua Er Bi Yan Hou Ke Za Zhi 38: 213-216, 2003 (In Chinese).

21. Ahuja A and Ying M: An overview of neck node sonography. Invest Radiol 37: 333-342, 2002.

22. Chikui T, Yonetsu K and Nakamura T: Multivariate feature analysis of sonographic findings of metastatic cervical lymph nodes: Contribution of blood flow features revealed by power Doppler sonography for predicting metastasis. AJNR Am J Neuroradiol 21: 561-567, 2000

23. Joo YH, Cho KJ, Kim SY and Kim MS: Prognostic significance of lymph node density in patients with hypopharyngeal squamous cell carcinoma. Ann Surg Oncol 22 (Suppl 3): S1014-S1019, 2015.

24. Roberts TJ, Colevas AD, Hara W, Holsinger FC, Oakley-Girvan I and Divi V: Number of positive nodes is superior to the lymph node ratio and American Joint Committee on Cancer N staging for the prognosis of surgically treated head and neck squamous cell carcinomas. Cancer 122: 1388-1397, 2016.

This work is licensed under a Creative Commons Attribution-NonCommercial-NoDerivatives 4.0 International (CC BY-NC-ND 4.0) License. 